\title{
Geographical Construction of Scale-Free Networks with Both Short Path Lengths and Hops
}

\author{
Yukio Hayashi ${ }^{1}$ and Jun Matsukubo ${ }^{2}$ \\ ${ }^{1}$ Japan Advanced Institute of Science and Technology, Ishikawa, Japan \\ ${ }^{2}$ Kitakyusyu National College of Technology, Fukuoka, Japan
}

\begin{abstract}
We find the structural effect in geographical networks on the optimal paths and on the robustness of the connectivity. The communication efficiency are measured by the average path lengths and hops in the typical planar networks: Delaunay triangulation, random Apollonian network, and our proposed model with the well-balanced properties. The dynamic configuration will be useful especially for ad hoc communication.
\end{abstract}

\section{Introduction}

Complex networks have been studied with great interest inspired from physics to biology, computer science, and other fields, since the surprisingly common topological structure called small-world (SW) or scale-free (SF) has been found in many real systems [1]. It has good properties in the meanings of economical and efficient communication by small number of hops in a connected network with a few links [2] and of the robustness against failure [3]. Moreover, the restriction of link lengths has been observed, e.g. Internet at both router and AS levels [4, road networks, and flight-connection in a major airline [5. Indeed, the distribution of link lengths was inversely proportional to the lengths [4] or exponentially decayed [6]. In other words, the length is not equal but various in a space, therefore some longrange links are more dominant to the shortest path whose distance is defined by a sum of the link lengths on the path between two nodes. This situation called disorder has been recently studied in complex networks [7, $[8$. On the other hand, although efficient transport of packets, passengers, supplies, or other quantities depends on both the topology and routing methods [9], there are local rules to find the optimal paths in a special class of networks such as planar graphs.

In this paper, we consider geographical SF network models for a number of research fields including urban planning, electric circuits, distributed robots, sensor networks, communication networks, and so on. In particular, we discuss dynamic configuration of planar networks for ad hoc communication. The planarity is important to avoid interference of the wireless beam, or to construct communication lines on the surface of earth.

In the state-of-the-art studies, a few geographical SF network models have been known with theoretical analyses in the evolutional mechanisms of powerlaw behavior. They are categorized into three classes: the modulated BarabásiAlbert (BA) model by a penalty of the distance for connecting nodes [10, [11, SF 
networks embedded in lattices [12, 13], and space-filing networks [14, 15], 16]. A brief review of these models has been reported [6. Unfortunately, crossing of links exist except for the third class. On the other hand, as a typical model in the third class, a random Apollonian network (RA) has some long-range links which cause dissipation of the beam power or the construction cost of links, although it is based on planar triangulation without crossing of links. Thus, to reduce long-range links, we consider a modification of RA preserving the good properties of SF structure on a planar space. We investigate the shortest paths with weak disorder and find the universal scaling laws. Moreover, the robustness against the attacks on hubs is improved.

\section{Models of Ad Hoc Networks}

\subsection{Planar Triangulation}

Planar triangulation is a mathematical abstraction of ad hoc networks, in which the positions of nodes are temporarily fixed as base stations of backbone networks. Thus, the mobility of node is out of our scope to simplify the discussion. On such graphs, online routing algorithms [17] that guarantee delivery of messages using only local information about positions of the source, destination, and the adjacent nodes to a current node in the routing have been developed. To find a path through exploration is required in many cases, since knowledge about the environment in which routing takes place is not available beforehand, especially in dynamic configurations with evolution. In any case, the optimal path depends on both topological and spatial network structures, as measures of the communication efficiency, the number of hops for transfer of a message and the path length in the Euclidean distance are crucial.

On the other hand, Delaunay triangulation (DT), which is the dual of a Voronoi diagram, is the optimal planar triangulation in some geometric criteria [18. with respect to the maximin angle and the minimax circumcircle of triangle on a two-dimensional space, and widely used in practical applications for facility locations and computer graphics [19]. It is well-known as a good property that the shortest path length between any two nodes on a Delaunay graph is of the same order as the direct Euclidean distance, since the ratio of the path length to the direct distance is bounded by a constant 20. However, the average number of hops on that graph is unknown. One of the fundamental techniques for equipping such properties is diagonal flipping. In the Delaunay triangulation, diagonal flips are globally applied to the triangles until the minimum angle of triangle is not increased by the exchange of diagonal links in a quadrilateral. Such global process is unsuitable for ad hoc networks. In contrast, RA can be constructed by local procedures for the subdivision of a randomly chosen triangle, although it has some long-range links. Thus, we compare the communication efficiency measured by the average path length and hops in the typical network models based on planar triangulation: DT in computer science, RA in complex network science, and our modification to bridge them. 


\subsection{Delaunay-Like SF Network}

We briefly explain a random Apollonian network (RA) 14 constructed from an initial triangulation of a polygon as follows. At each time step, a triangle is randomly chosen, and a new node is added inside the triangle and linked to its three nodes. We assume the new node is set at the barycenter of the chosen triangle. The topological properties of power-law degree distribution, large clustering coefficient, disassortative degree-degree correlation, and the average small number of the minimum hops on paths between any two nodes have been theoretically and numerically analyzed [14, 15, ,16. Although RAs have the several advanced SF properties and the SW effect with a small diameter of graph, some long-range links naturally appear near the boundary edges. To reduce the long-range links, we propose a modified model from RAs. The main idea is based on a strategy for connecting nodes in distances as short as possible by adding with the diagonal flips in DTs. The proposed network is grown as follows.

Step 0: Set an initial planar triangulation in a space.

Step 1: Select a triangle at random and add a new node at the barycenter. Then, connect the new node to its three nodes. Moreover, by iteratively applying diagonal flips, connect it to the nearest node (or more than one of the neighbor nodes) within a radius defined by the distance between the new node and the nearest node of the chosen triangle.

Step 2: The above process is repeated until the required size $N$ is reached.

We have two variations with one nearest node and all neighbors in the local circle, whereas the diagonal flips are globally applied in DTs. Note that these nodes are limited to the connected ones by applying iterative diagonal flips. We call our model $\mathrm{RA}+\mathrm{NN}$ (one/all) that means the combination with the triangulation in RAs and the rewiring to the one or all Nearest Neighbors denoted in the parentheses.

Fig. 1]illustrates the linking procedures by iterative diagonal flips: in a quadrilateral that consists of the shaded triangles, the long-range (cross) link is diagonally exchanged to the red link for maximizing the minimum angle of triangle. The dashed lines are new links from the barycenter, and form new five triangles with contours in the left of Fig. 1] The intersected black solid links with dashed ones are removed after the 2 nd flips. Thus, the concentrate of links to hubs is
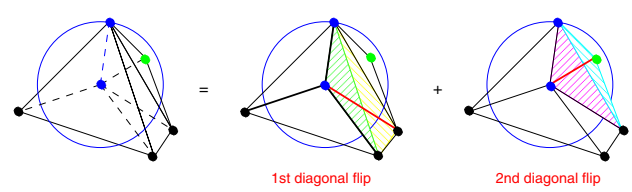

Fig. 1. Linking procedures in a Delaunay-like SF network. The intersected lines are exclusive in each shaded quadrilateral. The green node is the nearest from the barycenter.
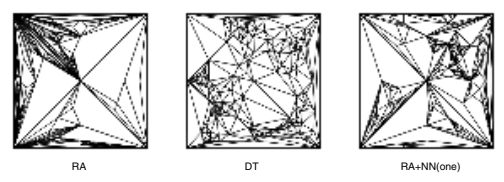

Fig. 2. Examples of RA: random Apollonian network, DT: Delaunay triangulation, and $\mathrm{RA}+\mathrm{NN}$ : our proposed model 
relaxed from connecting star-like stubs. Fig. 2 shows the topological characteristic that our RA+NN(one)s have the intermediate structure between those of RAs and DTs. The case of all neighbors is the same with the one's property.

\section{Efficiency for Communication}

\subsection{Approximative Analysis of the Degree Distribution}

The degree distribution is one of the important statistical characteristics related to the SF properties. We approximately derive the exponential decaying in the tail of degree distribution for the proposed network model. The occurrence of cutoffs also observed in real networks [21] is rather natural from the constraint on addition of new links.

When some links are removed from a node by multiple diagonal flips as shown in Fig. 1 the dynamical equation of the number of nodes $n(k, N)$ with degree $k$ at the size $N$ is given by

$$
n(k+1, N+1)=\frac{k}{N_{\Delta}} n(k, N)+\left(1-\frac{k+1}{N_{\Delta}}\right) n(k+1, N)-a \frac{k}{N_{\Delta}} n(k+1, N),
$$

where $N_{\Delta}$ and $a$ denotes the number of triangles and the average rate of the multiple diagonal flips, respectively. The 1st and 2nd terms in the r.h.s correspond to the preferential attachment through random selection of a triangle, and the 3rd term is the statistical rewiring effect by multiple diagonal flips. Note that there is no other reason for decreasing the degree. We neglect the other effects such as additional links to nodes with low degrees, because we focus on the tail of degree distribution.

By using $P(k)=n(k, N) / N$, we have

$$
\frac{N_{\Delta}+N}{N} P(k+1)+k(P(k+1)-P(k))+a k P(k+1)=0 .
$$

From the continuous approximation $d p / d k \approx P(k+1)-P(k)$ and $\gamma \stackrel{\text { def }}{=}\left(N_{\Delta}+\right.$ $N) / N$, it is rewritten as

$$
k \frac{d p}{d k}=-(\gamma+a k) p .
$$

Thus, we obtain the solution $p(k) \sim k^{-\gamma} \exp (-a k)$ for large $N$. Note that in RAs a power law with the exponent $\gamma_{R A} \approx 3$ has been similarly derived [14].

Fig. 31(a) shows the agreement of our approximation with the observed degree distribution. The cumulative rate $a$ is numerically estimated by the average convergent value as shown in Fig. 3(b). Note that the time step $t$ is equivalent to the network size $N$, because a new node is added at each time. Each network model is investigated in the averaging of 100 random realizations at the size $N=1,000$ generated from the initial triangulation of a square graph. 


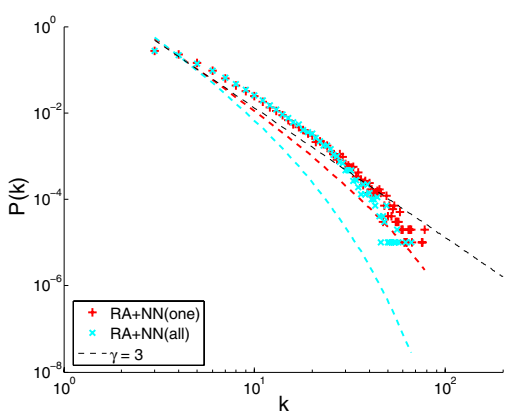

(a)

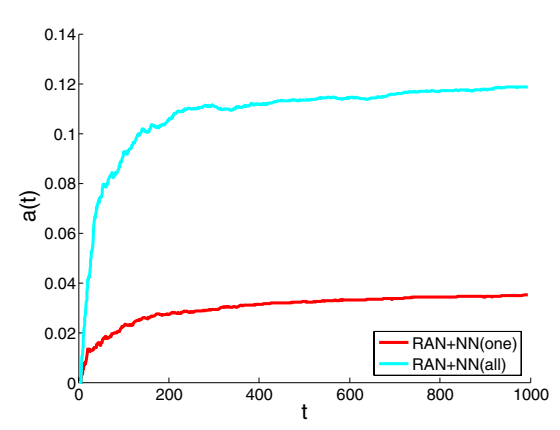

(b)

Fig. 3. Numerical estimation of (a) the power-law degree distribution with exponential cutoff. The red and cyan dashed lines show the approximations in the form $k^{-\gamma} \exp (-a k)$ with $\gamma=\left(N_{\Delta}+N\right) / N \approx 2.994, a=0.03$ for RA+NN(one), and $a=0.12$ for $\mathrm{RA}+\mathrm{NN}($ all). The black dashed line guides the slope of 3 . (b) the cumulative rate $a(t)$ of diagonal flips as a function of time step $t$.

\subsection{Weak Disorder in the Distribution of Link Lengths}

In the studies of the optimal path in disordered complex networks [7], [8], each link length is associated with a weight assumed by $\exp (\delta \varepsilon)$, where the parameter $\delta$ controls the strength of disorder, and $\varepsilon$ is a random number taken form a uniform distribution between 0 and 1 . As a network approaches the strong disorder limit at $\delta \rightarrow \infty$, only the longest link becomes dominant in the shortest path length defined by the smallest sum of link lengths on a path between two nodes. At the limit, the scaling relations of the average shortest path length $\langle D\rangle \sim N^{1 / 3}$ for $\gamma>4$ and $\langle D\rangle \sim N^{(\gamma-3) /(\gamma-1)}$ for $3<\gamma \leq 4$ has been theoretically predicted [7] from the percolation on SF networks [22]. Although the relation is unknown for $2<\gamma \leq 3$ because of the singularity in the analysis at $\gamma=3,\langle D\rangle \sim(\ln N)^{\gamma-1}$ has been also numerically suggested [7].

However, the assumption of length distribution may be violated on a geometric space, in addition the strong disorder limit is an extreme case. Thus, to investigate the strength of disorder in RAs, DTs, and the proposed networks, we compare the length distributions. Fig. 4 shows the distribution $P\left(l_{i j}\right)$ of link length $l_{i j}$ in each network. The dashed lines with an equal gap from top to bottom are corresponded to the distributions of weight $2 \exp (\delta \varepsilon) / \exp (\delta)$ for $\delta=1,2,4,8,16$, respectively. The factor $2 / \exp (\delta)$ is due to the normalization for the maximum length of the boundary edge. We find that RAs and RA+NN(one/all)s have weak disorder with small $\delta[\underline{8}$, while DTs have a slightly broad range of disorder as similar to the exponential decay in the domestic airline flight-connection [6].

\subsection{Shortest Path Lengths and Minimum Hops}

We investigate the average distance of path length $\langle D\rangle$ on the shortest paths, the distance $\left\langle D^{\prime}\right\rangle$ on the paths of the minimum hops, the average number of hops 


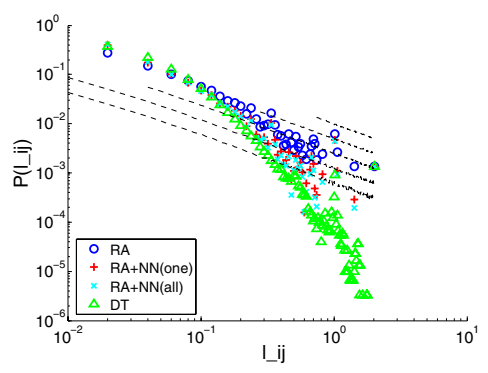

Fig. 4. The distribution of link lengths with weak disorder
Table 1. Estimated values of the exponents in the forms $\langle D\rangle \sim(\ln N)^{\beta_{d}},\left\langle D^{\prime}\right\rangle \sim(\ln N)^{\beta_{d^{\prime}}}$, $\langle L\rangle \sim(\ln N)^{\beta_{l}},\left\langle L^{\prime}\right\rangle \sim(\ln N)^{\beta_{l^{\prime}}},\langle L\rangle \sim N^{\alpha_{l}}$, $\left\langle L^{\prime}\right\rangle \sim N^{\alpha_{l^{\prime}}}$, by the mean-square-error method for each network

\begin{tabular}{c|cc|cc|cc}
\hline network model & $\beta_{d}$ & $\beta_{d}^{\prime}$ & $\alpha_{l}$ & $\alpha_{l}^{\prime}$ & $\beta_{l}$ & $\beta_{l}^{\prime}$ \\
\hline RA & 0.012 & -0.039 & 0.121 & 0.136 & 0.920 & 1.036 \\
DT & -0.068 & 0.416 & 0.332 & 0.455 & 2.525 & 3.452 \\
RA+NN(one) & -0.080 & 0.151 & 0.213 & 0.341 & 1.622 & 2.587 \\
RA+NN(all) & -0.106 & 0.320 & 0.216 & 0.346 & 1.641 & 2.628 \\
\hline
\end{tabular}

$\langle L\rangle$ on these paths, and the number of hops $\left\langle L^{\prime}\right\rangle$ on the shortest paths between any two nodes. Figs. [5(a)(b) show the SW effect. Note that the shortest path and the path of the minimum hops may be distinct, these measures are related to the link cost or delay and the load for transfer of a message. It is better to shorten both the distance and the number of hops, however their constraints are generally conflicted.

As in Table 1, we find the scaling relations estimated by straight lines for the sizes $N=500,1,000,3,000,5,000$ and 10,000. We remark that the values of $\beta_{d}$ and $\beta_{d^{\prime}}$ differ from $\gamma-1 \approx 2$ numerically suggested at the strong disorder limit [7, although the values of $\beta_{l}$ and $\beta_{l^{\prime}}$ are relatively close to it. The weak disorder may affect these differences. In addition, the values of $\alpha_{l}$ and $\alpha_{l^{\prime}}$ are close to 1/3 predicted at the limit 7] for the Erdös-Rényi (ER) model as the classical random network and the Watts-Strogatz (WS) model as a SW network. The nearest $\alpha_{l}$ in DT is probably caused by that the lognormal degree distribution resembles the unimodal shapes in ER and WS models rather than a power-law.

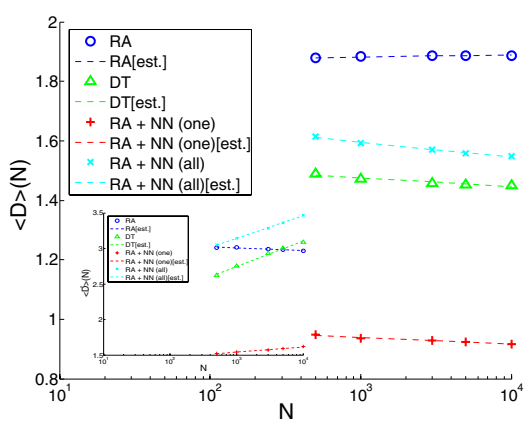

(a)

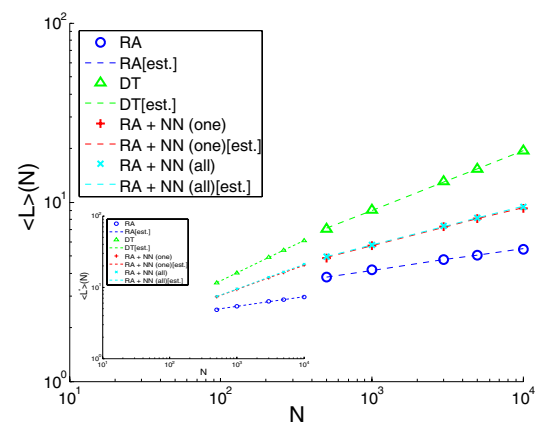

(b)

Fig. 5. Average distances and hops. Insets: the results on the exchanged paths. The dashed lines correspond the estimations of $\langle D\rangle \sim(\ln N)^{\beta_{d}}$ and $\langle L\rangle \sim N^{\alpha_{l}}$. 


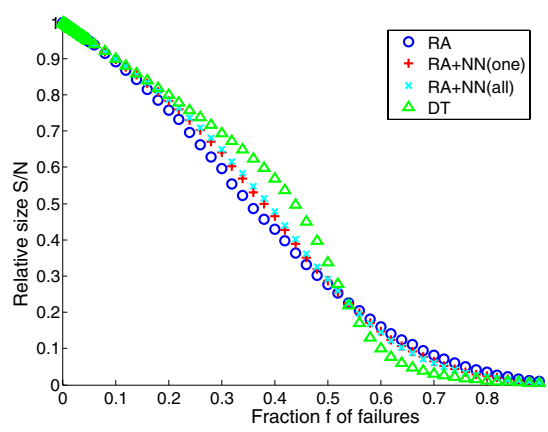

(a)

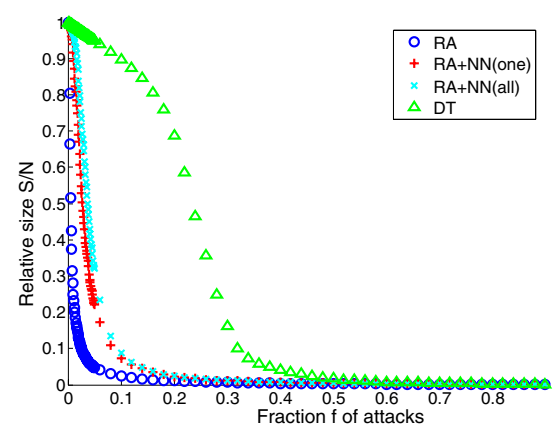

(b)

Fig. 6. Relative sizes $S / N$ of the giant component against (a) random failures and (b) attacks on hubs with the fraction $f$ of removed nodes. All networks have the same average degree $\langle k\rangle=2(3 N-7) / N=5.986$ and the minimum $k_{\min }=3$.

\section{Tolerance to Failure or Attack}

The fault tolerance and attack vulnerability are known as the typical SF properties 3. We compare the tolerance of connectivity in the giant component (GC) of RAs, DTs, and RA+NN(one/all)s, when a small fraction $f$ of the nodes is removed. Fig. 6(a) shows the similar results of the relative size $S / N$ for the fraction of random failures, where $S$ denotes the size of GC. Against the attacks on hubs selected in the decreasing order of degrees, Fig. 6(b) shows the improvements in $\mathrm{RA}+\mathrm{NN}$ (one/all)s from the extremely vulnerable RAs caused at an early stage by the disconnection of star-like stubs concentrated at the four corners and the center nodes as shown in Fig. 2, Note that the weakly inhomogeneous DT is different from a homogeneous random network, which has the same behavior against the failure and the attack at a fraction of removed nodes [3].

\section{Conclusion}

We study the communication efficiency of geographical networks called RA [14, 15] and DT [18, [19] based on planar triangulation for ad hoc networks [17]. In particular, to reduce long-range links, we propose a modified model whose degree distribution follows a power-law with exponential cutoff. We find a weak disorder in the distributions of link lengths. Even in the weak disorder, we suggest the scaling laws of the shortest path length $\langle D\rangle \sim(\ln N)^{\beta_{d}}$ and of the minimum hop $\langle L\rangle \sim N^{\alpha_{l}}$ as similar to the case at the strong disorder limit [7]. From the simulation results, we conclude that RAs have a path connected by a few hops but the path length becomes long including some long-range links, while DTs have a zig-zag path connected by many hops but each link is short. Instead of the superior geometric properties [18, DTs are no longer optimal in this criteria of the minimum hops. Our model is totally balanced: the shortest 
path length is the best, in addition the number of hops is the intermediate between ones in DTs and RAs. Moreover, by the structural effect, the robustness against the attacks on hubs is improved than that in RAs. Although DTs are the most robust in these network models, only it requires global configuration procedures that is unsuitable for ad hoc communication.

\section{References}

1. A.-L. Barabási, Linked: The New Science of Networks. Perseus (2002).

2. R.F.i Cancho, and R.V. Solé, Optimization in Complex Networks. In: R. PastorSatorras, M. Rubi, and A. Diaz-Guilera (eds): Statistical Mechanics in Complex Networks, Lecture Notes in Physics, Vol.625. Springer, Berlin (2003) 114-126.

3. R. Albert, and A.-L. Barabási, Nature, Vol.406, 378, (2000).

4. S.-H. Yook, H. Jeong, and A.-L. Barabási, PNAS, Vol.99(21),13382, (2002).

5. M.T. Gastner, and M.E.J. Newman, arXiv:cond-mat/0407680, (2004).

6. Y. Hayashi, IPSJ Journal, Special Issue on Network Ecology, Vol.47(3), (2006) or arXiv:physics/0512011, (2005).

7. L.A. Braunstein, et al., Phys. Rev. Lett., Vol.91, 168701, (2003).

8. T. Kalisky, et al., Phys. Rev. E, Vol.72, 025102, (2005).

9. B. Tadic, and S. Thurner, Physica A, Vol.332, 566, (2004) and 346, 183, (2005).

10. S.S. Manna, and S. Parongama, Phys. Rev. E, Vol.66, 066114, (2002).

11. R. Xulvi-Brunet, and I.M. Sokolov, Phys. Rev. E, Vol.66, 026118, (2002).

12. C.P. Warren, L.M. Sander, and I.M. Sokolov, Phys. Rev. E, Vol.66, 056105, (2002).

13. D. ben-Avraham, et al., Physica A, Vol.330, 107, (2003).

14. T. Zhou, G. Yan, and B.-H. Wang, Phys. Rev. E, Vol.71, 046141, (2005).

15. J.P.K. Doye, and C.P. Massen, Phys. Rev. E, Vol.71, 016128, (2004).

16. J.S. Andrade,Jr., et al., Phys. Rev. Lett., Vol.94, 018702, (2005).

17. P. Bose, and P. Morin, SIAM J. of Computing, Vol.33(4), 937, (2004).

18. K. Imai, IEICE Trans. on Infor. and Syst., Vol.83-D(3), 428, (2000).

19. A. Okabe, et al., Spatial Tessellations, 2nd ed. John Wiley (2000).

20. J.M. Keil, and C.A. Gutwin, Discrete Compt. Geom., Vol.7, 13, (1992)

21. L.A.N. Amaral, et al., PNAS, Vol.97(21), 11149, (2000).

22. R Cohen et al., Structural properties of scale-free networks. In: S. Bornholdts, and H.G. Shuster (eds): Handbook of Graphs and Networks. Wiely-VCH, New York (2002) 85-110. 